\title{
Carotid artery plaque assessment using quantitative expansive remodeling evaluation and MRI plaque signal intensity
}

\author{
Yoshitaka Kurosaki, MD, ${ }^{1}$ Kazumichi Yoshida, MD, PhD, ${ }^{2}$ Ryu Fukumitsu, MD, ${ }^{2}$ \\ Nobutake Sadamasa, MD, PhD, ${ }^{1}$ Akira Handa, MD, PhD, ${ }^{1}$ Masaki Chin, MD, ${ }^{1}$ and \\ Sen Yamagata, MD, PhD'
}

'Department of Neurosurgery, Kurashiki Central Hospital, Okayama; and ${ }^{2}$ Department of Neurosurgery, Kyoto University School of Medicine, Kyoto, Japan

\begin{abstract}
OBJECTIVE Plaque characteristics and morphology are important indicators of plaque vulnerability. MRI-detected intraplaque hemorrhage has a great effect on plaque vulnerability. Expansive remodeling, which has been considered compensatory enlargement of the arterial wall in the progression of atherosclerosis, is one of the criteria of vulnerable plaque in the coronary circulation. The purpose of this study was risk stratification of carotid artery plaque through the evaluation of quantitative expansive remodeling and MRI plaque signal intensity.

METHODS Both preoperative carotid artery T1-weighted axial and long-axis MR images of 70 patients who underwent carotid endarterectomy (CEA) or carotid artery stenting (CAS) were studied. The expansive remodeling ratio (ERR) was calculated from the ratio of the linear diameter of the artery at the thickest segment of the plaque to the diameter of the artery on the long-axis image. Relative plaque signal intensity (rSI) was also calculated from the axial image, and the patients were grouped as follows: Group $A=r S I \geq 1.40$ and ERR $\geq 1.66$; Group $B=r S I<1.40$ and ERR $\geq 1.66$; Group $C=r S I \geq 1.40$ and ERR $<1.66$; and Group $D=r S I<1.40$ and ERR $<1.66$. Ischemic events within 6 months were retrospectively evaluated in each group.
\end{abstract}

RESULTS Of the 70 patients, 17 (74\%) in Group A, $6(43 \%)$ in Group B, 7 (44\%) in Group C, and $6(35 \%)$ in Group D had ischemic events. Ischemic events were significantly more common in Group $A$ than in Group $D(p=0.01)$.

CONCLUSIONS In the present series of patients with carotid artery stenosis scheduled for CEA or CAS, patients with plaque with a high degree of expansion of the vessel and T1 high signal intensity were at higher risk of ischemic events. The combined assessment of plaque characterization with MRI and morphological evaluation using ERR might be useful in risk stratification for carotid lesions, which should be validated by a prospective, randomized study of asymptomatic patients.

http://thejns.org/doi/abs/10.3171/2015.2.JNS142783

KEY WORDS carotid artery MRI; carotid artery disease; expansive remodeling; intraplaque hemorrhage; risk stratification; vascular disorders

$\mathrm{C}$ AROTID artery atherosclerosis is one of the important causes of ischemic stroke. The efficacy of carotid endarterectomy (CEA) for severe symptomatic stenosis of the carotid artery has been demonstrated in several multicenter, randomized, clinical trials. ${ }^{7,8,18}$ Currently, the therapeutic approach for preventing future ischemic events, including CEA, carotid artery stenting (CAS), and medical treatment, is decided primarily based on the percentage of luminal narrowing of the vessel. However, it has been shown that thromboembolic mechanisms correlate strongly with ischemic events in carotid artery stenosis. ${ }^{16,36}$ Thus, a method for evaluating the risk of thromboembolism might improve the ability to identify the actual high-risk patients who would benefit most from intervention. In recent studies, some investigators have reported that plaque with a lipid-rich necrotic core

ABBREVIATIONS BB = black blood; CAS = carotid artery stenting; $C E=$ contrast enhanced; $C E A=$ carotid endarterectomy; ERR = expansive remodeling ratio; ICA = internal carotid artery; IPH = intraplaque hemorrhage; $r S I=$ relative plaque signal intensity; VD = vessel diameter.

SUBMITTED December 8, 2014. ACCEPTED February 3, 2015.

INCLUDE WHEN CITING Published online September 11, 2015; DOI: 10.3171/2015.2.JNS142783. 
and intraplaque hemorrhage (IPH), one of the features of vulnerable plaque, can be detected as high signal intensity on carotid T1-weighted MRI ${ }^{10,35,37}$ and is strongly associated with ipsilateral ischemic stroke. ${ }^{1,14}$ Other investigators have shown that geometrical change of the artery correlates with the risk of ischemic events, and that expansive remodeling may be particularly associated with plaque instability and a high risk for ischemic events. ${ }^{12}$ This study aimed to provide more accurate risk stratification of carotid plaque through the evaluation of quantitative expansive remodeling and MRI plaque signal intensity.

\section{Methods}

\section{Patient Population}

A total of 89 consecutive carotid stenoses in 86 patients who were scheduled to undergo CEA or CAS due to atherosclerotic carotid stenosis were included in this study. Patients who did not undergo either carotid T1-weighted axial or long-axis MR images, had poor imaging quality, had near occlusion, or had a history of CEA on the ipsilateral side were excluded. Inclusion criteria for revascularization were $\geq 70 \%$ carotid stenosis or $<70 \%$ symptomatic stenosis with recurrent infarcts in the ipsilateral hemisphere refractory to maximal medical therapy.

Patient characteristics were recorded retrospectively by reviewing medical records. Ischemic events ipsilateral to the carotid artery within the previous 6 months were recorded, including cerebral infarction, transient ischemic attack, and retinal ischemia (amaurosis fugax and retinal artery occlusion). The severity of carotid stenosis was evaluated by digital subtraction angiography using the North American Symptomatic Trial (NASCET) collaborators' criteria. ${ }^{18}$ The hospital ethics committee approved the study, and written informed consent was obtained from all patients.

\section{Imaging Techniques}

Carotid artery MRI was performed using a 1.5-T MRI machine (Gyroscan Intera; Philips Medical Systems) equipped with an 8-cm-diameter surface coil. A previously published standardized protocol was used to obtain black-blood (BB) T1-weighted axial and long-axis images of the carotid arteries, including the area with the highest stenosis on the index side. ${ }^{33,35}$ The parameters for the imaging sequences were as follows: 1) long-axis T1-weighted images (3D inversion recovery [IR] turbo field echo): TR $10 \mathrm{msec}$; TE $2.7 \mathrm{msec}$; inversion time (TI) $500 \mathrm{msec}$; flip angle (FA) $35^{\circ} ; 320 \times 512$ matrix; 1.6 -mm section thickness; 150-mm FOV; 2) axial T1-weighted images (2D double IR-turbo spin-echo images): TR 700-1000 msec; TE 12 msec; FA $110^{\circ}$; $256 \times 256$ matrix; 3-mm section thickness; 150-mm FOV; and TR, 1 cardiac cycle. Fat suppression was used to reduce signals from subcutaneous fat. All patients underwent BB-MRI within 2 weeks before surgery.

\section{MRI Signal Intensity}

Signal intensities of plaque and the proximal sternocleidomastoid muscle on T1-weighted axial MR images were measured at a workstation by a colleague (K.Y. or R.F.) who was blinded to patient information. Relative MRI signal intensity was calculated with the following formula (Fig. 1): ${ }^{35} \mathrm{rSI}=$ SI whole plaque/SI SCM, in which rSI represents relative plaque signal intensity, SI represents signal intensity, and SCM represents sternocleidomastoid muscle. With reference to the previous study, ${ }^{35}$ an $\mathrm{rSI} \geq 1.40$, which corresponds to the lipid core with IPH, was defined as hyperintense plaque.

\section{Expansive Remodeling Ratio}

We have previously reported about the expansive remodeling ratio (ERR). ${ }^{34}$ The measurement technique for the ERR was developed by applying the method used in NASCET to evaluate stenosis. ${ }^{18}$ The ERR was calculated using long-axis BB-MRI and the following formula (Fig. 2): $\mathrm{ERR}=$ the maximum distance between the lumen and the outer borders of the plaque perpendicular to the axis of the internal carotid artery (ICA) (stenotic ICA vessel diameter [VD]/the maximal luminal diameter of the distal ICA at a region unaffected by atherosclerosis [distal ICA VD]).

In our previous study about the ERR, the control ERR measured in the contralateral nonatherosclerotic ICA in patients with unilateral carotid artery stenosis was reported. ${ }^{34}$ The control ERR (mean \pm SD) was $1.36 \pm 0.15$. Two SDs of the mean control ERR, 1.66 or more, was defined as high ERR.

\section{Relationships Between MRI Signal Intensity, ERR, and Symptoms}

Based on whether patients had hyperintense plaque and high ERR, the patients were grouped as follows: Group A $=\mathrm{rSI} \geq 1.40$ and $\mathrm{ERR} \geq 1.66$; Group $\mathrm{B}=\mathrm{rSI}<1.40$ and

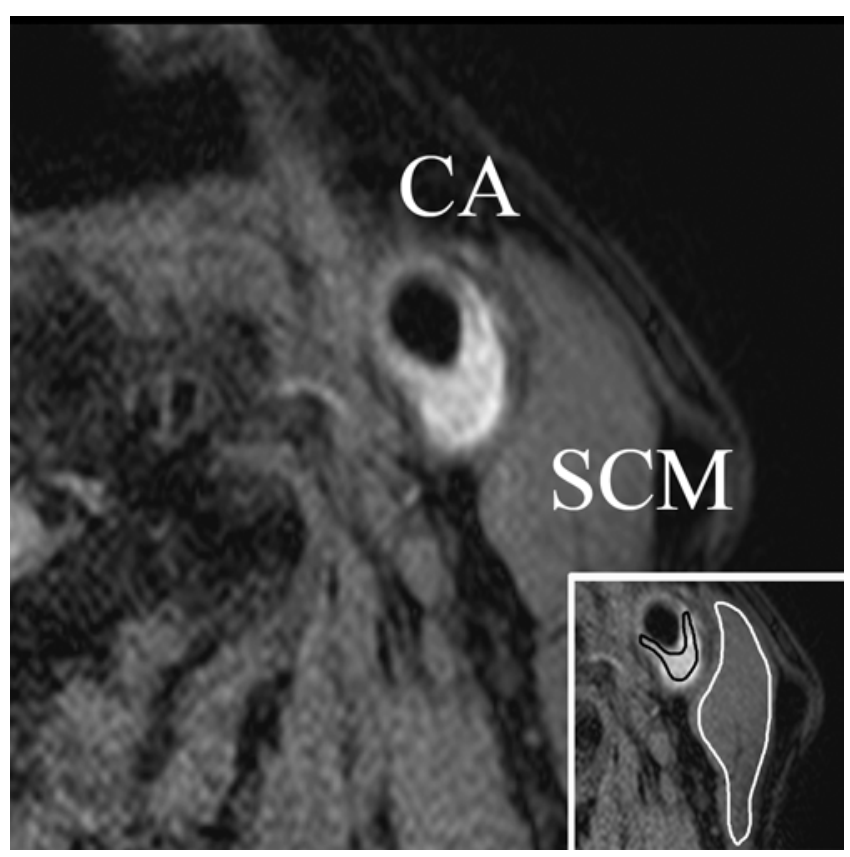

FIG. 1. Measurement of $r S I$ in the carotid artery $(C A)$ using axial, highresolution MRI. The rSI is measured with the region of interest drawn over the whole plaque except for the lumen with reference to the sternocleidomastoid muscle (SCM). The rSI in this symptomatic patient is 1.73 . 


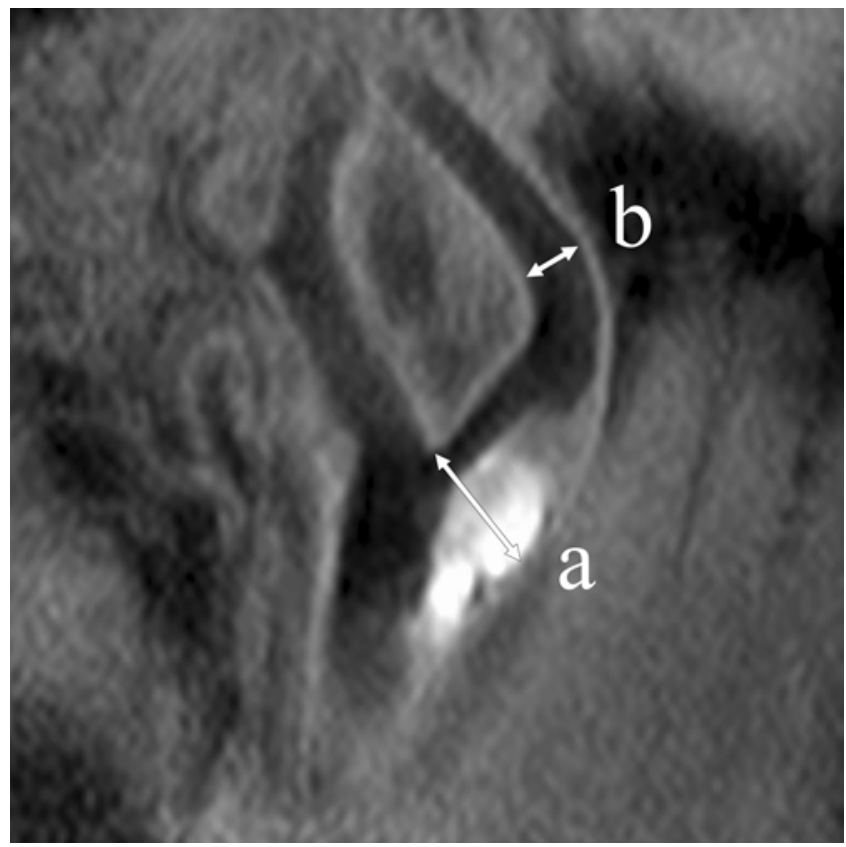

FIG. 2. Measurement of the ERR in the carotid artery using long-axis, high-resolution MRI. The ERR is calculated as the ratio of the maximal outside diameter of the atherosclerotic ICA near the carotid bulb (a) and the maximal outside diameter of the ICA well beyond the plaque (b). The ERR in this symptomatic patient is 2.79 .

ERR $\geq 1.66$; Group $\mathrm{C}=\mathrm{rSI} \geq 1.40$ and $\mathrm{ERR}<1.66$; and Group $\mathrm{D}=\mathrm{rSI}<1.40$ and $\mathrm{ERR}<1$.66. Ipsilateral ischemic events within 6 months were retrospectively evaluated in each group.

\section{Statistical Analysis}

All statistical analyses were performed using SPSS for Windows (version 17.0, SPSS Inc.). Differences in the age of the baseline characteristics of each group were analyzed by the Kruskal-Wallis test, while differences in sex and risk factors were analyzed using the chi-square for independence test. The differences in the stenosis rate, rSI, and ERR between groups were analyzed using the Kruskal-Wallis test, using 1-way ANOVA for the distal ICA VD and stenotic ICA VD. The chi-square test for independence was used to assess the relationship between group and ischemic events within 6 months. The level of significance was established at $\mathrm{p}<0.05$.

\section{Results}

Of the 89 patients, 8 did not undergo MRI, 3 had studies of poor imaging quality, 5 had near occlusion, 2 had restenosis after $\mathrm{CEA}$, and 1 had plaque confirmed in the common carotid artery, leaving 70 patients for evaluation. Of these 70 patients, Group A had 23 patients, Group B had 14, Group C had 16, and Group D had 17 (Fig. 3). Except for hypertension, no significant differences existed in baseline characteristics among the groups (Table 1).

The stenosis rate was $65.1 \% \pm 25.1 \%$ in Group A, $81.7 \%$ $\pm 9.4 \%$ in Group B, $66.8 \% \pm 17.6 \%$ in Group C, and $74.5 \%$ $\pm 11.5 \%$ in Group D (Table 2). The stenosis rate differed significantly among the 4 groups, with the lowest rate in
Group A. The rSI was $1.68 \pm 0.24$ in Group A; $1.22 \pm 0.10$ in Group B; $1.69 \pm 0.21$ in Group C; and $1.14 \pm 0.15$ in Group D. The rSI also differed significantly among the 4 groups. The ERR was $2.14 \pm 0.31$ in Group A; $1.96 \pm 0.19$ in Group B; $1.43 \pm 0.16$ in Group $C$; and $1.41 \pm 0.98$ in Group D. The ERR also differed significantly among the 4 groups (Table 2 ).

The relationship between group and patients with an ipsilateral ischemic event within 6 months was examined. The number of patients with ipsilateral ischemic events within 6 months was $17(73 \%)$ in Group A, $6(43 \%)$ in Group B, 7 (44\%) in Group C, and $6(35 \%)$ in Group D. There were significantly more ipsilateral ischemic events in Group A than in Group D $(\mathrm{p}=0.01)$, and the number of patients with ipsilateral ischemic events was relatively higher in Group A than in Groups B $(\mathrm{p}=0.058)$ and C (p $=0.056$; Fig. 4 ).

\section{Discussion}

The results of the present study demonstrated a relationship between the extent of expansive remodeling and plaque signal intensity rSI and the presence of symptoms. In the present study, patients with a high degree of expansion of the vessel, a T1 high-signal-intensity plaque, and IPH were more likely to have ischemic events within 6 months. These results indicate the potential contribution of the combined assessment of morphological evaluation using ERR and plaque characterization with rSI for the prediction of a patient's risk for a future stroke. Patients with higher ERR and IPH may be at high risk for cerebral infarction. The combined assessment of ERR and plaque signal intensity rSI could be useful in risk stratification for carotid lesions.

Recent vascular biology studies have indicated that plaque vulnerability is an important risk factor for ischemic events. ${ }^{3}$ Various evaluation methods for plaque vulnerability have been reported:11,23,28 evaluation using signal intensity on carotid artery MRI is also used in clinical practice ${ }^{14,25}$ and is useful for identification of high-risk lesions. ${ }^{13,19}$ Recent technological developments in MRI equipment and imaging sequencing make it possible to assess the carotid plaque components. ${ }^{35}$ In particular, IPH, which is a characteristic component of vulnerable plaque, was detected as high signal intensity on T1-weighted imaging, and it confers higher risk for ischemic events than its absence in both symptomatic ${ }^{1}$ and asymptomatic ${ }^{29}$ patients. However, plaque vulnerability depends on not only the plaque components, but also other factors, such as plaque volume and plaque morphology. ${ }^{17}$ Therefore, more accurate risk assessment would be possible by adding evaluation of these factors.

Expansive vascular remodeling is a morphological change in the artery ${ }^{9}$ and has been considered one of the criteria for vulnerable plaque in the coronary arteries. ${ }^{20}$ Plaque with expansive remodeling has been shown to have a significantly larger lipid core and higher macrophage count than negatively or less positively remodeled plaque. ${ }^{31}$ Recent studies have also shown that expansive carotid remodeling was associated with low endothelial stress and plaque rupture ${ }^{4,5}$ and was significantly greater in patients 


\section{IC stenosis CEA or CAS $(n=89)$}

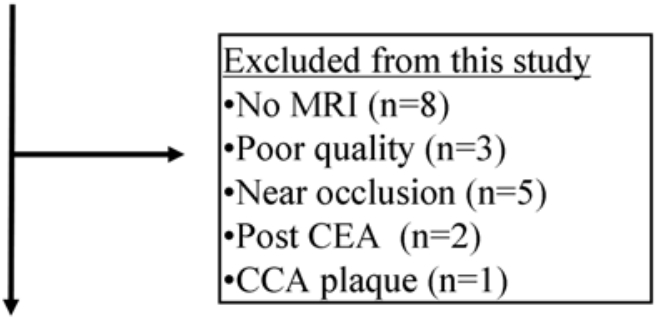

\section{0 patients}

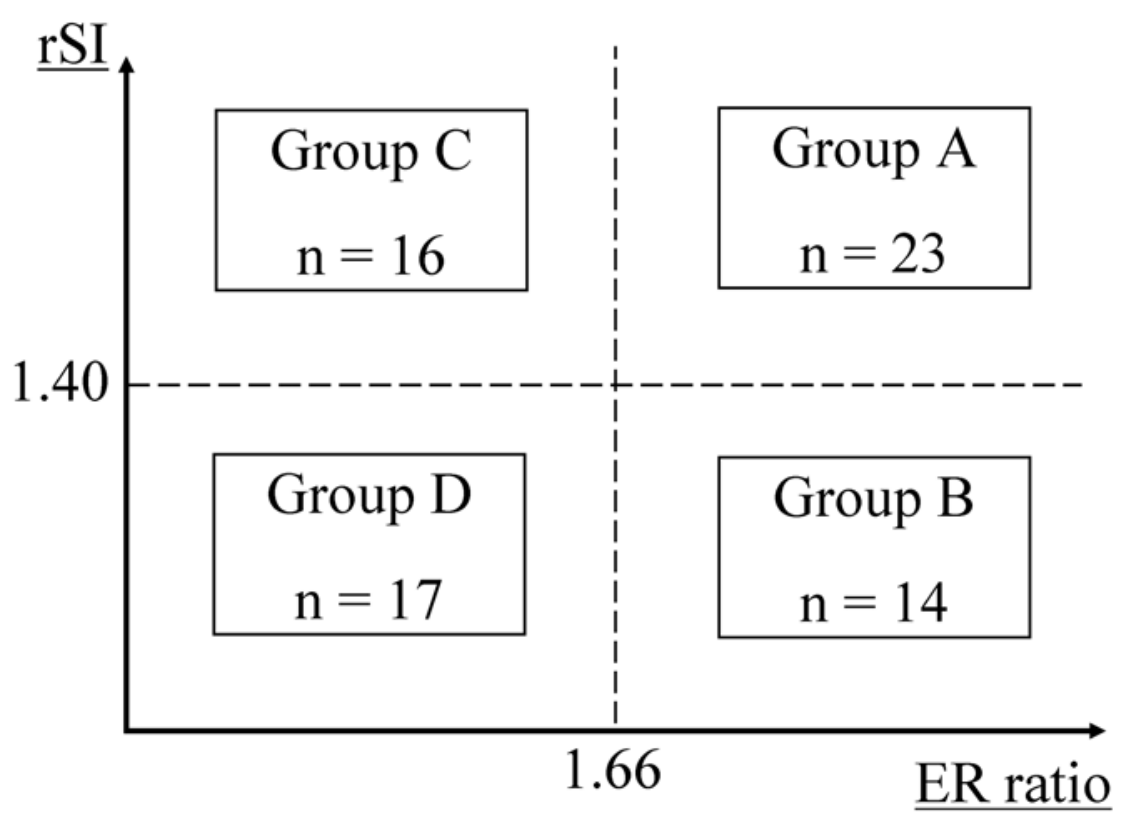

FIG. 3. Grouping based on rSI and ERR.

with cerebral ischemic symptoms than in asymptomatic patients. ${ }^{12}$ Therefore, we hypothesize that evaluation with plaque signal intensity and expansive remodeling is useful for identifying higher-risk lesions. In fact, in the present series of patients scheduled for CEA and CAS, ischemic

TABLE 1. Patient characteristics by group*

\begin{tabular}{lccccc}
\hline \multicolumn{1}{c}{ Characteristic } & Group A & Group B & Group C & Group D & p Value \\
\hline No. of patients & \multicolumn{1}{c}{23} & \multicolumn{1}{c}{14} & \multicolumn{1}{c}{16} & 17 & \\
\hline Mean age (yrs) & 74.4 & \multicolumn{1}{c}{72.9} & 72.7 & 72.2 & 0.3 \\
\hline Females & $3(13)$ & $3(21)$ & $1(6)$ & $1(5)$ & 0.4 \\
\hline Hypertension & $22(95)$ & $10(71)$ & $13(81)$ & $17(100)$ & 0.04 \\
\hline Diabetes mellitus & $7(30)$ & $2(14)$ & $6(37)$ & $4(23)$ & 0.5 \\
\hline Hyperlipidemia & $14(60)$ & $5(35)$ & $10(62)$ & $12(70)$ & 0.2 \\
\hline Smoking & $6(26)$ & $6(42)$ & $9(56)$ & $8(47)$ & 0.2 \\
\hline $\begin{array}{l}\text { Ischemic heart } \\
\text { disease }\end{array}$ & $7(30)$ & $4(28)$ & $3(18)$ & $7(41)$ & 0.5 \\
\hline
\end{tabular}

* Data are shown as number (\%) of patients unless otherwise indicated. Group $A=r S I \geq 1.40$ and ERR $\geq 1.66$; Group B $=r S I<1.40$ and ERR $\geq 1.66$; Group $C=r S I \geq 1.40$ and $E R R<1.66$; and Group $D=r S I<1.40$ and ERR $<1.66$. events within 6 months were significantly higher in patients with IPH and expansive remodeling than in patients without IPH and expansive remodeling.

Concerning factors related to vulnerability of carotid plaque, other important markers are plaque cap thickness and inflammation. ${ }^{17}$ The fibrous cap is a layer of connective tissue separating the lipid-rich necrotic core (which includes IPH) of the atherosclerotic plaque from the carotid artery lumen. With rupture of fibrous caps, plaque components are exposed to flowing blood, which may result in arterial thrombus formation, thus leading to ischemic events. ${ }^{27} \mathrm{~A}$ thin or ruptured fibrous cap is the strongest risk factor for ipsilateral ischemic events. ${ }^{8}$ Thus, an assessment method that includes evaluation of fibrous cap status may enable more accurate risk stratification. Noninvasive options including contrast-enhanced (CE) MRI, ${ }^{2}$ ultrasonography, ${ }^{6}$ and $\mathrm{CT}^{32}$ have been investigated to assess fibrous cap integrity, but inadequate spatial resolution remains a problem. A combination of minimum cap thickness $<200 \mu \mathrm{m}$ and a representative cap thickness $<500 \mu \mathrm{m}$ identified ruptured plaques most reliably, ${ }^{21}$ but spatial resolution of CE MRI is $390 \times 390 \mu \mathrm{m}$ and in-plane resolutions of ultrasonography and CT are 300 
TABLE 2. rSI and ERR by group*

\begin{tabular}{lccccc}
\hline \multicolumn{1}{c}{ Variable } & Group A & Group B & Group C & Group D & p Value† \\
\hline No. of patients & 23 & 14 & 16 & 17 & \\
\hline Stenosis rate (\%) & $65.1 \pm 25.1$ & $81.7 \pm 9.4$ & $66.8 \pm 17.6$ & $74.5 \pm 11.5$ & 0.03 \\
\hline rSI & $1.68 \pm 0.24$ & $1.22 \pm 0.10$ & $1.69 \pm 0.21$ & $1.14 \pm 0.19$ & $<0.001$ \\
\hline ERR & $2.14 \pm 0.31$ & $1.96 \pm 0.19$ & $1.43 \pm 0.16$ & $1.41 \pm 0.15$ & $<0.001$ \\
\hline Distal ICA VD $(\mathrm{mm})$ & $4.07 \pm 0.66$ & $4.02 \pm 0.80$ & $4.64 \pm 0.56$ & $4.87 \pm 0.98$ & 0.002 \\
\hline Stenotic ICA VD $(\mathrm{mm})$ & $8.64 \pm 1.27$ & $7.95 \pm 1.99$ & $6.63 \pm 1.08$ & $6.83 \pm 1.19$ & $<0.001$ \\
\hline
\end{tabular}

* Values are presented as the mean \pm SD.

$\dagger$ Boldface values are statistically significant ( $p \leq 0.05)$.

$\times 400 \mu \mathrm{m}$ and $500 \times 500 \mu \mathrm{m}$, respectively. ${ }^{15}$ Improved diagnostic technology offers the hope of more accurate prediction of carotid artery disease risk.

Inflammation of plaque may also play an important role in plaque vulnerability. ${ }^{22}$ Inflamed plaques may readily cause artery-to-artery thromboembolism through plaque rupture..$^{15}$ Noninvasive options, including CE MRI ${ }^{26}$ and 18F-fluorodeoxyglucose (FDG) PET, ${ }^{30}$ have been investigated to assess the degree of inflammation. Tawakol et al. ${ }^{30}$ demonstrated that FDG-PET can determine the macrophage content of carotid plaques in vivo. Furthermore, others demonstrated that a combination of FDG-PET and MRI is complementary in identifying high-risk carotid plaques with inflamed or hemorrhagic components. ${ }^{24}$ FDG-PET may be useful for noninvasively identifying macrophage-rich carotid plaques. Furthermore, its combination with carotid artery MRI may provide more useful information on plaque composition. However, it would be difficult to routinely perform a dual PET/MRI examination because of cost. In addition, dual PET/MRI needs longer imaging time, because it involves different kinds of modalities. On the other hand, the present assessment of ERR and rSI can be obtained simply and rapidly from the same modality, carotid BB-MRI. Therefore, this combination method is a good candidate for routine imaging

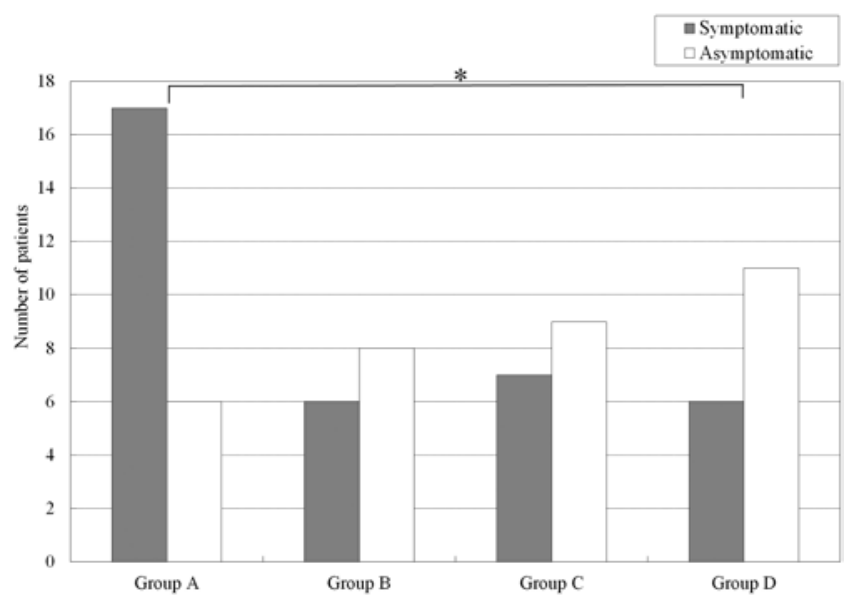

FIG. 4. Relationships among the 4 groups with respect to the numbers of patients with ipsilateral ischemic events within 6 months. The number of patients with ipsilateral ischemic events was significantly higher in Group $A$ than in Group $D(p=0.01)$, and it was relatively higher in Group A than in Groups $B(p=0.058)$ and $C(p=0.056)$. evaluation of carotid disease, especially for asymptomatic patients, and it may make it possible to select truly nonstenotic high-risk lesions such as mild carotid stenosis with expansive remodeling and high-volume plaque, ${ }^{36}$ which would not be identified for intervention by the current stenosis-based method.

\section{Limitations of the Study}

The limitations of this study include its small sample size and its retrospective nature. Some selection bias may have been introduced by having study patients who were scheduled to have CEA or CAS. Therefore, it appears that patients with a higher ERR and greater hyperintensity were more likely to have reported symptoms prior to revascularization, but this does not mean that expansive remodeling or rSI can be used to predict future events in an entirely asymptomatic and unselected population. In addition, because most studies about the relationship between expansive remodeling and ischemic events were retrospective, it is not clear that expansive remodeling truly causes ischemic events. A plaque with a high ERR in a symptomatic patient could merely represent fresh IPH. It is possible that expansive remodeling could be the result of rapid growth of IPH at the ischemic event. However, in the present study, even with IPH, the number of symptomatic plaques in Group A, accompanied by high ERR, was higher than that in Group C, which was not accompanied by high ERR. This result might demonstrate the potential predictive value of the ERR for ischemic events.

Next, one possible limitation is the threshold of ERR. A high value was defined as 2 standard deviations (1.66) or more of the mean control ERR (1.36), which was calculated from normal carotid arteries without stenosis in our previous study. ${ }^{34}$ In the same previous study, the mean ERR of patients with carotid stenosis was $1.68 \pm 0.40$, and the receiver-operating characteristic analysis found that when the cutoff value of ERR was set at 1.88, the sensitivity and specificity for the detection of ischemic symptom were 0.6 and 0.78 , respectively. If the cutoff value of ERR was set at 1.88 in the present study, the number of patients with ischemic events within 6 months was also significantly higher in Group A (rSI $\geq 1.4$ and ERR $\geq 1.88$ ) than in Group D (rSI $<1.4$ and ERR $<1.88 ; \mathrm{p}=0.01)$. Thus, assessment of dual plaque signal intensity and quantitative remodeling evaluation may be reasonable, but the cutoff value of ERR remains to be elucidated. To investigate the usefulness of the combined assessment of ERR 
and plaque signal intensity for accurate stroke risk stratification, a long-term prospective study, including asymptomatic patients with early-stage carotid atherosclerosis, should be performed.

\section{Conclusions}

In the present series of carotid artery plaque scheduled for CEA or CAS, plaque with a high degree of expansion of the vessel and T1 high signal intensity was at higher risk of causing ischemic events. These findings suggest that the combination of quantitative expansive remodeling evaluation and rSI with MRI might noninvasively contribute to more accurate risk stratification, but this does not mean that expansive remodeling or plaque signal intensity can be used to predict future events in an entirely asymptomatic and unselected population. To investigate the predictive value of ERR and plaque signal intensity for future ischemic events, a prospective study, including asymptomatic patients with early-stage carotid atherosclerosis, should be performed.

\section{References}

1. Altaf N, Daniels L, Morgan PS, Auer D, MacSweeney ST, Moody AR, et al: Detection of intraplaque hemorrhage by magnetic resonance imaging in symptomatic patients with mild to moderate carotid stenosis predicts recurrent neurological events. J Vasc Surg 47:337-342, 2008

2. Cai J, Hatsukami TS, Ferguson MS, Kerwin WS, Saam T, Chu B, et al: In vivo quantitative measurement of intact fibrous cap and lipid-rich necrotic core size in atherosclerotic carotid plaque: comparison of high-resolution, contrast-enhanced magnetic resonance imaging and histology. Circulation 112:3437-3444, 2005

3. Carr S, Farb A, Pearce WH, Virmani R, Yao JS: Atherosclerotic plaque rupture in symptomatic carotid artery stenosis. J Vasc Surg 23:755-766, 1996

4. Chatzizisis YS, Coskun AU, Jonas M, Edelman ER, Feldman CL, Stone PH: Role of endothelial shear stress in the natural history of coronary atherosclerosis and vascular remodeling: molecular, cellular, and vascular behavior. J Am Coll Cardiol 49:2379-2393, 2007

5. Chatzizisis YS, Jonas M, Coskun AU, Beigel R, Stone BV, Maynard C, et al: Prediction of the localization of high-risk coronary atherosclerotic plaques on the basis of low endothelial shear stress: an intravascular ultrasound and histopathology natural history study. Circulation 117:993-1002, 2008

6. Devuyst G, Karapanayiotides T, Ruchat P, Pusztaszeri M, Lobrinus JA, Jonasson L, et al: Ultrasound measurement of the fibrous cap in symptomatic and asymptomatic atheromatous carotid plaques. Circulation 111:2776-2782, 2005

7. European Carotid Surgery Trialists' Collaborative Group: Randomised trial of endarterectomy for recently symptomatic carotid stenosis: final results of the MRC European Carotid Surgery Trial (ECST). Lancet 351:1379-1387, 1998

8. Executive Committee for the Asymptomatic Carotid Atherosclerosis Study: Endarterectomy for asymptomatic carotid artery stenosis. JAMA 273:1421-1428, 1995

9. Glagov S, Weisenberg E, Zarins CK, Stankunavicius R, Kolettis GJ: Compensatory enlargement of human atherosclerotic coronary arteries. N Engl J Med 316:1371-1375, 1987

10. Görtler M, Goldmann A, Mohr W, Widder B: Tissue characterisation of atherosclerotic carotid plaques by MRI. Neuroradiology 37:631-635, 1995

11. Grønholdt ML, Nordestgaard BG, Schroeder TV, Vorstrup $\mathrm{S}$, Sillesen $\mathrm{H}$ : Ultrasonic echolucent carotid plaques predict future strokes. Circulation 104:68-73, 2001
12. Hardie AD, Kramer CM, Raghavan P, Baskurt E, Nandalur KR: The impact of expansive arterial remodeling on clinical presentation in carotid artery disease: a multidetector CT angiography study. AJNR Am J Neuroradiol 28:1067-1070, 2007

13. U-King-Im JM, Young V, Gillard JH: Carotid-artery imaging in the diagnosis and management of patients at risk of stroke. Lancet Neurol 8:569-580, 2009

14. Kurosaki Y, Yoshida K, Endo H, Chin M, Yamagata S: Association between carotid atherosclerosis plaque with high signal intensity on T1-weighted imaging and subsequent ipsilateral ischemic events. Neurosurgery 68:62-67, 2011

15. Kwee RM, van Engelshoven JM, Mess WH, ter Berg JW, Schreuder FH, Franke CL, et al: Reproducibility of fibrous cap status assessment of carotid artery plaques by contrastenhanced MRI. Stroke 40:3017-3021, 2009

16. Moustafa RR, Izquierdo-Garcia D, Fryer TD, Graves MJ, Rudd JH, Gillard JH, et al: Carotid plaque inflammation is associated with cerebral microembolism in patients with recent transient ischemic attack or stroke: a pilot study. Circ Cardiovasc Imaging 3:536-541, 2010

17. Naghavi M, Libby P, Falk E, Casscells SW, Litovsky S, Rumberger J, et al: From vulnerable plaque to vulnerable patient: a call for new definitions and risk assessment strategies: Part I. Circulation 108: 1664-1672, 2003

18. North American Symptomatic Carotid Endarterectomy Trial Collaborators: Beneficial effect of carotid endarterectomy in symptomatic patients with high-grade carotid stenosis. $\mathbf{N}$ Engl J Med 325:445-453, 1991

19. Parmar JP, Rogers WJ, Mugler JP III, Baskurt E, Altes TA, Nandalur KR, et al: Magnetic resonance imaging of carotid atherosclerotic plaque in clinically suspected acute transient ischemic attack and acute ischemic stroke. Circulation 122:2031-2038, 2010

20. Pasterkamp G, Galis ZS, de Kleijn DP: Expansive arterial remodeling: location, location, location. Arterioscler Thromb Vasc Biol 24:650-657, 2004

21. Redgrave JN, Gallagher P, Lovett JK, Rothwell PM: Critical cap thickness and rupture in symptomatic carotid plaques: the oxford plaque study. Stroke 39:1722-1729, 2008

22. Ross R: Atherosclerosis-an inflammatory disease. N Engl J Med 340:115-126, 1999

23. Rudd JH, Warburton EA, Fryer TD, Jones HA, Clark JC, Antoun N, et al: Imaging atherosclerotic plaque inflammation with $[18 \mathrm{~F}]$-fluorodeoxyglucose positron emission tomography. Circulation 105:2708-2711, 2002

24. Saito H, Kuroda S, Hirata K, Magota K, Shiga T, Tamaki N, et al: Validity of dual MRI and F-FDG PET imaging in predicting vulnerable and inflamed carotid plaque. Cerebrovasc Dis 35:370-377, 2013

25. Sakamoto M, Taoka T, Nakagawa H, Takayama K, Wada T, Myouchin K, et al: Magnetic resonance plaque imaging to predict the occurrence of the slow-flow phenomenon in carotid artery stenting procedures. Neuroradiology 52:275-283, 2010

26. Schmitz SA, Coupland SE, Gust R, Winterhalter S, Wagner S, Kresse M, et al: Superparamagnetic iron oxide-enhanced MRI of atherosclerotic plaques in Watanabe hereditable hyperlipidemic rabbits. Invest Radiol 35:460-471, 2000

27. Spagnoli LG, Mauriello A, Sangiorgi G, Fratoni S, Bonanno E, Schwartz RS, et al: Extracranial thrombotically active carotid plaque as a risk factor for ischemic stroke. JAMA 292:1845-1852, 2004

28. Streifler JY, Eliasziw M, Fox AJ, Benavente OR, Hachinski VC, Ferguson GG, et al: Angiographic detection of carotid plaque ulceration. Comparison with surgical observations in a multicenter study. North American Symptomatic Carotid Endarterectomy Trial. Stroke 25:1130-1132, 1994

29. Takaya N, Yuan C, Chu B, Saam T, Underhill H, Cai J, et al: 
Association between carotid plaque characteristics and subsequent ischemic cerebrovascular events: a prospective assessment with MRI-initial results. Stroke 37:818-823, 2006

30. Tawakol A, Migrino RQ, Bashian GG, Bedri S, Vermylen $\mathrm{D}$, Cury RC, et al: In vivo $18 \mathrm{~F}$-fluorodeoxyglucose positron emission tomography imaging provides a noninvasive measure of carotid plaque inflammation in patients. J Am Coll Cardiol 48:1818-1824, 2006

31. Varnava AM, Mills PG, Davies MJ: Relationship between coronary artery remodeling and plaque vulnerability. Circulation 105:939-943, 2002

32. Wintermark M, Jawadi SS, Rapp JH, Tihan T, Tong E, Glidden DV, et al: High-resolution CT imaging of carotid artery atherosclerotic plaques. AJNR Am J Neuroradiol 29:875882,2008

33. Yoshida K, Endo H, Sadamasa N, Narumi O, Chin M, Inoue $\mathrm{K}$, et al: Evaluation of carotid artery atherosclerotic plaque distribution by using long-axis high-resolution black-blood magnetic resonance imaging. J Neurosurg 109:1042-1048, 2008

34. Yoshida K, Fukumitsu R, Kurosaki Y, Funaki T, Kikuchi T, Takahashi JC, et al: The association between expansive remodeling detected by high-resolution MRI in carotid stenosis and clinical presentation. J Neurosurg 123:434-440, 2015

35. Yoshida K, Narumi O, Chin M, Inoue K, Tabuchi T, Oda K, et al: Characterization of carotid atherosclerosis and detection of soft plaque with use of black-blood MR imaging. AJNR Am J Neuroradiol 29:868-874, 2008

36. Yoshida K, Sadamasa N, Narumi O, Chin M, Yamagata S,
Miyamoto S: Symptomatic low-grade carotid stenosis with intraplaque hemorrhage and expansive arterial remodeling is associated with a high relapse rate refractory to medical treatment. Neurosurgery 70:1143-1151, 2012

37. Yuan C, Mitsumori LM, Beach KW, Maravilla KR: Carotid atherosclerotic plaque: noninvasive MR characterization and identification of vulnerable lesions. Radiology 221:285-299, 2001

\section{Disclosure}

The authors report no conflict of interest concerning the materials or methods used in this study or the findings specified in this paper.

\section{Author Contributions}

Conception and design: Kurosaki. Acquisition of data: Kurosaki, Yoshida, Fukumitsu, Handa. Analysis and interpretation of data: Kurosaki. Drafting the article: Kurosaki. Critically revising the article: Yoshida. Reviewed submitted version of manuscript: Yoshida, Sadamasa, Chin. Study supervision: Sadamasa, Chin, Yamagata.

\section{Correspondence}

Yoshitaka Kurosaki, Department of Neurosurgery, Kurashiki Central Hospital, 1-1-1 Miwa, Kurashiki City, Okayama 7108602, Japan. email: kurosaki0106@gmail.com. 